\title{
Thermodynamics of QCD from Sakai-Sugimoto model
}

\author{
Hiroshi Isono,,$^{a, b}$ Gautam Mandal ${ }^{c}$ and Takeshi Morita ${ }^{d}$ \\ ${ }^{a}$ Department of Physics, National Tsing Hua University, \\ Hsinchu 30013, Taiwan \\ ${ }^{b}$ Department of Physics, Particle Physics Research Laboratory, Faculty of Science, \\ Chulalongkorn University, Phayathai Road, Patumwan, Bangkok 10330, Thailand \\ ${ }^{c}$ Department of Theoretical Physics, Tata Institute of Fundamental Research, \\ Mumbai 400 005, India \\ ${ }^{d}$ Department of Physics, Shizuoka University, \\ 836 Ohya, Suruga-ku, Shizuoka 422-8529, Japan \\ E-mail: hiroshi.isono81@gmail.com, mandal@theory.tifr.res.in, \\ morita.takeshi@shizuoka.ac.jp
}

ABSTRACT: Till date, the only consistent description of the deconfinement phase of the Sakai-Sugimoto model appears to be provided by the analysis of [1]. The current version of the analysis, however, has a subtlety regarding the monodromy of quarks around the Euclidean time circle. In this note, we revisit and resolve this issue by considering the effect of an imaginary baryon chemical potential on quark monodromies. With this ingredient, the proposal of [1] for investigating finite temperature QCD using holography is firmly established. Additionally, our technique allows a holographic computation of the free energy as a function of the imaginary chemical potential in the deconfinement phase; we show that our result agrees with the corresponding formula obtained from perturbative QCD, namely the Roberge-Weiss potential.

KEYwords: Gauge-gravity correspondence, Holography and quark-gluon plasmas, Phase Diagram of QCD

ARXIV EPRINT: 1507.08949 


\section{Contents}

1 Introduction 1

2 Deconfinement phase in Sakai-Sugimoto model and the issue of quark monodromy

$3 \quad$ Imaginary baryon chemical potential and quark monodromy 5

4 The imaginary chemical potential in the Sakai-Sugimoto model $\quad 6$

$\begin{array}{lll}5 & \text { Free energy as a function of imaginary chemical potential } & 7\end{array}$

$\begin{array}{ll}\text { A A review of imaginary chemical potentials in field theory } & \mathbf{1 0}\end{array}$

A.1 Relation between imaginary chemical potential and periodicity of fermions 10

A.2 Relation between imaginary chemical potential and gauge fields 11

$\begin{array}{lll}\text { A.3 Derivation of (4.1) } & 12\end{array}$

A.4 Periodicity of thermal partition function with imaginary chemical potential 12

$\begin{array}{lll}\mathrm{B} \mathrm{Z}_{N_{c}} \text { symmetry for } \boldsymbol{\theta} \text { in gravity } & 13\end{array}$

\section{Introduction}

The Witten-Sakai-Sugimoto model $[2,3]$ has been of profound significance in investigating QCD using holography [4]. Witten's model for a holographic dual of pure Yang-Mills theory [2] involved essentially $N_{c}$ D4 branes on $\mathbf{R}^{3} \times$ a Scherk-Schwarz circle. Sakai and Sugimoto [3] added external fundamental quarks to this model by adding $N_{f}$ D8 and $\overline{\mathrm{D} 8}-$ branes as probes and demonstrated that this model explains the low energy dynamics of QCD such as chiral symmetry breaking and the meson spectrum. By now, a large body of literature has grown (see, e.g. $[5,6]$ for recent developments) which successfully explains various qualitative aspects of QCD at strong coupling using this model.

It is, thus, natural to apply this model to systems at finite temperature. Such a study, indeed, was initiated by Aharony et al. [7], who, following [8], employed the black D4brane geometry as the gravity dual to the deconfinement phase in QCD and explained chiral symmetry restoration at high temperature. This was followed by many authors who investigated this model to explore various outstanding problems in thermal QCD such as the phase structure including the finite baryon chemical potential, which is difficult to compute in lattice gauge theory due to the well-known sign problem [9-14].

Ref. [7], however, also pointed out that the correspondence between the black D4brane geometry and the deconfinement phase in QCD is not without problems, since there must be at least one phase boundary between these two phases, which, therefore, are 
not smoothly connected. This issue was further explored by two of the present authors (MM) [1], who showed that the black D4-brane geometry cannot describe four dimensional QCD due to issues related to the large- $N_{c}$ volume independence $[15,16]$ (as applied to the Scherk-Schwarz circle). Instead, [1] proposed that the deconfinement phase in QCD is represented by a rather different geometry, involving a localized solitonic D3-brane. It was shown that the gravity solution and deconfined QCD belong to the same phase; in addition, [1] showed that the new geometry too offers a mechanism of chiral symmetry restoration $(\chi \mathrm{SR}){ }^{1}$

There have been many follow-up works on finite temperature QCD from holography which support the MM proposal. In particular, it is argued in [6] that the estimate of the deconfinement transition temperature obtained from MM fits phenomenology better than the one obtained from the Witten-Sakai-Sugimoto model $[2,3]$. The papers $[17,18]$ argue that an alternative description of the high temperature phase of QCD is possible in a more elaborate setup using D4, NS5 and D6, $\overline{\mathrm{D} 6}$ branes, although within the Sakai-Sugimoto setup the high temperature phase indeed follows MM. Besides, ref. [19] demonstrated the similarity between the confinement/deconfinement transition in the gauge theory and the phase transition in the MM model, and a related connection can be seen even analytically in supersymmetric Yang-Mills theory [20]. More recently, ref. [21] showed that the MM model qualitatively reproduces the instanton density in lattice QCD while the black D4brane geometry does not.

The MM construction of the deconfinement phase, however, has an unsolved question regarding the monodromy of quarks around the temporal circle; we will discuss this below in section 2. The purpose of this article is to resolve this issue and thus firmly establish the MM method [1] for investigating finite temperature QCD via holography. We achieve this resolution by considering, as already hinted at in MM, an imaginary baryon chemical potential [22] (see sections 3 and 4). We find that this leads to the correct monodromy of the quarks suitable for describing thermal physics. As an additional application of our technique, we compute in section 5 the dependence of the free energy on the imaginary chemical potential; we find that the result is consistent with the Roberge-Weiss potential [22] ${ }^{2}$ which is obtained from perturbative QCD at high temperature.

\section{Deconfinement phase in Sakai-Sugimoto model and the issue of quark monodromy}

To obtain $\mathrm{SU}\left(N_{c}\right)$ QCD with $N_{f}$ flavor quarks, Sakai and Sugimoto [3] considered the following brane configuration

\begin{tabular}{l|cccccccccc} 
& $(t)$ & 1 & 2 & 3 & $(4)$ & 5 & 6 & 7 & 8 & 9 \\
\hline$N_{c}$ D4-brane & - & - & - & - & - & & & & & \\
$N_{f}$ D8/D8-brane & - & - & - & - & & - & - & - & - & -
\end{tabular}

\footnotetext{
${ }^{1}$ Like in [7], here too $\chi \mathrm{SR}$ is preceded by the deconfinement transition, although unlike in [7], the latter is a Gregory-Laflamme transition.

${ }^{2}$ See, e.g., [23] for some recent progress on QCD with complex chemical potential.
} 
Here the parentheses denote directions compactified on $S^{1}$. To represent finite temperature, spacetime is regarded as Euclidean; $t$ parameterizes the Euclidean time circle with period $\beta=1 / T . x^{4}$ is the Scherk-Schwarz circle, with antiperiodic boundary condition for fermions, which is crucial to break supersymmetry and to obtain the (non-supersymmetric) four dimensional QCD at low energy [2]. We define the period of this circle to be $L_{4}$.

We will consider below $N_{f} \ll N_{c}$ so that we can treat the D8-branes as probes. In an appropriate large $N_{c}$ limit à la Maldacena $[4,24]$ we can replace the $\mathrm{D} 4$ branes by a classical geometry, and regard the D8 branes as probes coupled to such a geometry. At low temperatures, the geometry, which in fact is the dual gravity description to compactified 5 dimensional SYM theory [24], is given by

$$
\begin{aligned}
& d s^{2}=\alpha^{\prime}\left[\frac{u^{3 / 2}}{\sqrt{\lambda_{5} / 4 \pi}}\left(d t^{2}+\sum_{i=1}^{3} d x_{i}^{2}+f_{4}(u) d x_{4}^{2}\right)+\frac{\sqrt{\lambda_{5} / 4 \pi}}{u^{3 / 2}}\left(\frac{d u^{2}}{f_{4}(u)}+u^{2} d \Omega_{4}^{2}\right)\right], \\
& f_{4}(u)=1-\left(\frac{u_{0}}{u}\right)^{3}, \quad e^{\phi}=\frac{\lambda_{5}}{(2 \pi)^{2} N_{c}}\left(\frac{u^{3 / 2}}{\sqrt{\lambda_{5} / 4 \pi}}\right)^{1 / 2} .
\end{aligned}
$$

There is also a non-trivial value of the five form potential which we do not write explicitly. Here $\lambda_{5}:=(2 \pi)^{2} g_{s} l_{s} N_{c}$ is the 't Hooft coupling on the D4-brane world-volume. $g_{s}$ and $l_{s}=\sqrt{\alpha^{\prime}}$ are the string coupling and the string length, respectively. We will also use the dimensionless coupling $\lambda_{Y M}:=2 \lambda_{5} / L_{4}$. The identifications $t \equiv t+\beta, x_{4} \equiv x_{4}+L_{4}$ are implicit.

Since the $x_{4}$-cycle shrinks to zero at $u=u_{0}$, in order to avoid possible conical singularities we must choose $L_{4}$ as follows

$$
\frac{L_{4}}{2 \pi}=\frac{\sqrt{\lambda_{5} / 4 \pi}}{3} u_{0}^{-1 / 2}
$$

With this choice, the contractible $x_{4}$-cycle, together with the radial direction $u$, forms a so-called 'cigar' geometry which is topologically a disc.

Note that the above gravity solution is reliable in a regime $\lambda_{Y M} \gg 1$ in which the stringy corrections are suppressed. On the other hand in the regime $\lambda_{Y M} \ll 1$, the system flows to IR and the massive modes such as adjoint fermions (and scalars), together with KK modes about the $x^{4}$-circle are decoupled and we obtain four dimensional QCD [3]. ${ }^{3}$ It turns out, however, that although there is no direct overlap of validity between four dimensional QCD at weak coupling $\left(\lambda_{Y M} \ll 1\right)$ and gravity at strong coupling $\left(\lambda_{Y M} \gg 1\right)$, it has been a highly rewarding enterprise to understand QCD dynamics by extrapolation from the gravity analysis (see, e.g, $[5,6]$ for recent developments). We should note that such an extrapolation is analogous to the use of the strong coupling expansion in lattice gauge theory. An important consideration for the success of the latter expansion is that there should not be a phase boundary between the strong coupling theory and the continuum theory which is defined at zero lattice coupling [25]. The point of the MM proposal [1]

\footnotetext{
${ }^{3}$ The theory we obtain is actually four dimensional pure Yang Mills theory [2] with external quarks in the fundamental representation coming from the D4-D8 $(\overline{\mathrm{D} 8})$ open strings.
} 
was to ensure that such phase boundaries were not encountered between gravity and fourdimensional QCD either at low or at high temperature.

To investigate high temperature regime using the gravity dual in the sense mentioned above, [1] pointed out that it was imperative to impose the periodic boundary condition along the temporal circle for the fermions. ${ }^{4}$ As we increase the temperature, strings which wind the temporal circle become lighter, and the type IIA gravity analysis is not reliable when the temperature reaches around $T \sim \sqrt{\lambda_{Y M}} / L_{4}$. This leads us to a type IIB supergravity description obtained by performing a T-duality on the temporal circle. ${ }^{5}$ Then the analysis is reliable if $T \gg \sqrt{\lambda_{Y M}} / L_{4} \cdot{ }^{6}$ This T-duality maps the brane configuration of the Sakai-Sugimoto model (2.1) to

\begin{tabular}{|c|c|c|c|c|c|c|c|c|c|c|}
\hline & $\left(t^{\prime}\right)$ & 1 & 2 & 3 & (4) & 5 & 6 & 7 & 8 & 9 \\
\hline$N_{c}$ D3-brane & & - & - & - & - & & & & & \\
\hline$N_{f} \mathrm{D} 7 / \overline{\mathrm{D} 7}$-brane & & - & - & - & & - & - & - & - & - \\
\hline
\end{tabular}

Here $t^{\prime}$ is the dual temporal circle, and its periodicity is $\beta^{\prime}=(2 \pi)^{2} / \beta=(2 \pi)^{2} T$.

The IIB supergravity analysis shows that around

$$
T_{G L} \sim \frac{\lambda_{4}}{L_{4}}
$$

the T-dual of the gravity solution (2.2) becomes unstable due to the Gregory-Laflamme instability [26], and a Gregory-Laflamme transition occurs. The stable solution at higher temperatures is the localized solitonic D3-brane geometry. Generally the analysis of the localized geometry in a compact space is difficult; however, an approximate geometry of the localized solitonic D3-brane geometry, can be described by the metric [1]

$$
\begin{array}{rlrl}
d s^{2} & =\alpha^{\prime}\left[H^{-1 / 2}\left(\sum_{i=1}^{3} d x_{i}^{2}+(1+2 \Phi) d x_{4}^{2}\right)+H^{1 / 2}\left(1-\frac{1}{2} \Phi\right)\left(d u^{2}+d t^{\prime 2}+u^{2} d \Omega_{4}^{2}\right)\right] \\
H & =\sum_{n} \frac{2 \lambda_{5} / \beta}{\left(u^{2}+\left(t^{\prime}-t_{0}^{\prime}-n \beta^{\prime}\right)^{2}\right)^{2}}, & e^{\phi} & =\frac{\lambda_{5}}{2 \pi N_{c} \beta} \\
\Phi & =-\frac{u_{H}^{4}}{2} \sum_{n}\left(\frac{1}{u^{2}+\left(t^{\prime}-t_{0}^{\prime}-n \beta^{\prime}\right)^{2}}\right)^{2}, & u_{H} & =\sqrt{2 \lambda_{5} T} \frac{\pi}{2 L_{4}}
\end{array}
$$

\footnotetext{
${ }^{4}$ The usual antiperiodic boundary condition leads to the black D4 brane at high temperature, which is separated from four dimensional deconfined QCD by a phase boundary, whereas the use of the periodic boundary condition leads to a different gravity solution, as we will recall presently, which does not involve such a phase boundary. The consequence of this choice for the description of high temperature QCD is detailed at the end of this section, and is the main issue addressed in this paper.

${ }^{5}$ If we took the periodicity of the fermions along the temporal circle to be anti-periodic, the system would be mapped to type 0B rather than to type IIB.

${ }^{6}$ To be more precise, the mass of the winding string depends on its position, and the lightest one is located at $u=u_{0}$ in the IIA flame. However after the T-duality, since the radius is flipped, the heaviest one is from $u=u_{0}$ in the IIB flame. Thus, if $T \gg \sqrt{\lambda_{Y M}} / L_{4}$, while the IIA supergravity analysis around $u \sim u_{0}$ is not reliable, the IIB supergravity is, although the validity of the latter away from this region is not ensured. However, since it is only the region $u \sim u_{0}$ which is involved in exploring the confinement/deconfinement transition, the use of IIB supergravity in $T \gg \sqrt{\lambda_{Y M}} / L_{4}$ is justified.
} 
The approximation used here is valid in a region far from the localized D3-branes and for $T \gg u_{H}$. Note that this solution describes D3-branes localized around $t^{\prime}=t_{0}^{\prime}$ and it breaks the translation symmetry along the $t^{\prime}$-circle spontaneously. We fix $t_{0}^{\prime}=0$ in the following discussions. [1] argued that it is this localized D3-brane geometry that corresponds to the deconfinement phase in QCD, and the Gregory-Laflamme transition leading to this localized solution corresponds to the confinement/deconfinement transition. ${ }^{7}$

To study quarks in the deconfinement phase, we need to study the probe D7branes (2.4) on the background geometry (2.6). However, we appear to encounter a problem here. As we explained above, we have imposed a periodic spin structure (around the temporal circle) in our supergravity analysis. Naively, this would seem to suggest ${ }^{8}$ periodic boundary condition on all QCD fermions, while the standard description of thermodynamics requires the boundary condition to be antiperiodic. The thermodynamics of the pure Yang-Mills sector is not affected by this problem, since at weak coupling, the adjoint fermions on the D3-branes (D4-branes in the IIA description) are anyway decoupled from the theory, hence the choice of their temporal boundary condition is immaterial [1]. However, since the fundamental quarks of QCD in the Sakai-Sugimoto model are not decoupled in this limit, a periodic temporal boundary condition for them would appear to be at variance with standard rules of QCD thermodynamics. The resolution of the above problem is a central issue of this paper.

\section{Imaginary baryon chemical potential and quark monodromy}

In this section, we consider the issue of monodromy of fermions in QFT in somewhat more general terms. Let us suppose that the quarks $\psi$ in four dimensional $\mathrm{SU}\left(N_{c}\right)$ QCD satisfy the following boundary condition around the temporal circle ${ }^{9}$

$$
\psi(t+\beta)=-e^{-i \theta} \psi(t) .
$$

As shown in appendix A, a path integral with the above boundary condition can be interpreted as a thermal partition function with an imaginary baryon chemical potential $(\mu=i \theta)$

$$
Z(\beta, \theta):=\operatorname{tr}\left(e^{-\beta \hat{H}+i \theta \hat{N}}\right)
$$

Here $\hat{N}$ is the baryon number operator. In appendix A and what follows, we show how such a chemical potential arises from in the presence of a thermal Wilson line or, equivalently, from the temporal component a constant $\mathrm{U}(1)$ external gauge potential. We will indicate

\footnotetext{
${ }^{7}$ The spontaneous symmetry breaking of the translation symmetry corresponds to the breaking of the center symmetry in the deconfinement phase.

${ }^{8}$ Strictly speaking, the periodic spin structure in gravity refers to gravitinos and other bulk fermions. To connect it to the spin structure of the gauge theory, we need to consider the AdS/CFT dictionary which relates gauge invariant boundary fermion operators to bulk fermions. The naive assumption referred to here is that the spin structure of the gauge theory is simply the same as that of supergravity. As we will see below, in our model they differ by an imaginary baryon chemical potential.

${ }^{9}$ The temporal circle is Euclidean and is labelled by $t$. Note that $\theta=\pi$ corresponds to the periodic boundary condition and $\theta=0$ to the anti-periodic one.
} 
in the next section, how such a gauge potential is automatically present in our problem (it is determined by the location of the D7 brane on the temporal circle). We will find that

(a) Choosing the above parameter (location of the D7 brane) judiciously, we can obtain a thermal partition function starting from a path integral over periodic fermions (section 4). This resolves the issue of apparent conflict between periodic fermion boundary condition and thermodynamics.

(b) Keeping this parameter arbitrary, the holographic setup allows us to determine the dependence of the free energy as a function of the imaginary chemical potential (section 5). The computation agrees with the corresponding computation from perturbative QCD - a result called the the Roberge-Weiss potential.

\section{The imaginary chemical potential in the Sakai-Sugimoto model}

We will now show how an imaginary baryon chemical potential such as in (3.2) presents itself in the Sakai-Sugimoto model in our context (see [27] for a related discussion). ${ }^{10}$ Let us first recall that the standard (real) chemical potential in Euclidean $\mathrm{SU}\left(N_{c}\right)$ QCD is equivalent to a constant pure-imaginary temporal component of the Euclideanized diagonal flavor gauge field. Hence an imaginary chemical potential is related to the real-valued Euclideanized flavor gauge field (see (A.2) for a precise relation between the two). In the Sakai-Sugimoto model, the flavor gauge field corresponds to a gauge field on D8/D8branes. Combining this observation with the fact that fermions on the string theory side are periodic in our setup, we find that a thermal partition function with an imaginary chemical potential $\theta$ can be regarded as a periodic functional integral with an external flavor gauge potential $A_{t}^{f}$ through

$$
\beta A_{t}^{f}=\theta+\pi
$$

Note that the factor $\pi$ comes from the difference of the periodicities. We give a detailed derivation of (4.1) in appendix A.3.

We discussed the imaginary chemical potential above (and in appendix A) directly in terms of quarks in the D4-D8 open strings. In the dual supergravity theory, the quarks are not present directly, but baryons are. It is easy to demonstrate how baryons pick up nontrivial monodromies around the thermal circle in the presence of a constant diagonal flavor gauge field. A baryon in the gravity dual is represented by a 'baryon vertex' (a D4-brane which wraps the $S^{4}$ of the geometry (2.2)) [3]. The D4-brane couples to the diagonal flavor gauge field (considered as external) through a Chern-Simons term as ${ }^{11}$

$$
\exp \left(i \int A \wedge F_{4}\right) \sim \exp \left(i N_{c} \int_{0}^{\beta} d t A_{t}^{f}\right)
$$

\footnotetext{
${ }^{10}$ Note that the imaginary chemical potential does not affect the periodicity of the color adjoint fermions and they remain periodic.

${ }^{11}$ The same result can be derived in the gauge theory on the D8-branes also, where the baryon realizes as an instanton [3].
} 
The gravity functional integral in the presence of this D4-brane defines the baryon wavefunction. As one takes $t \rightarrow t+\beta$, the functional integral, and hence the baryon wavefunction, picks up an extra phase $e^{i N_{c} \beta A_{t}^{f}}$ due to the presence of the flavor gauge field.

Thus, to introduce an arbitrary imaginary chemical potential, we need to simply turn on an appropriate constant, diagonal, flavor gauge field $A_{t}^{f}$, according to (4.1). To proceed further, we need to do a bit more work. (4.1) is written in terms of type IIA variables. As explained in section 2, to describe the high temperature limit in our holographic framework (corresponding to deconfined QCD) we need to T-dualize on the temporal circle. In that case, $2 \pi A_{t}^{f}$ is mapped to the position $t_{f}^{\prime},{ }^{12}$ of the $\mathrm{D} 7 / \overline{\mathrm{D} 7}$-branes on the dual temporal circle. The type IIB version of (4.1) is, then ${ }^{13}$

$$
t_{f}^{\prime}=\frac{2 \pi}{\beta}(\pi+\theta)=\frac{\beta^{\prime}}{2 \pi}(\pi+\theta)
$$

In particular, we can satisfy the above condition with (this was indeed the choice made in [1])

$$
t_{f}^{\prime}=\beta^{\prime} / 2 \quad(\Rightarrow \theta=0) .
$$

Recall that by convention we have put the D3 branes at $t^{\prime}=0$. Equation (4.4) implies that if the D7 $/ \overline{\mathrm{D} 7}$-branes are placed antipodally from the D3 branes on the temporal circle of the IIB theory (equivalent to a choice $\theta=0$ ), the path integral with periodic fermions does reduce to a thermal partition function, thus resolving the apparent puzzle raised in section 2 .

One might wonder whether we can place the D7 branes at will at any point on the temporal circle. In case the D7 branes are infinitely heavy, the position of the D7's can be regarded as an external parameter. However, in the next section, we will consider the potential energy of the D7 branes, placed at a position $t_{f}^{\prime}$ at the boundary $(u \rightarrow \infty) .{ }^{14}$ We will find that the choice $\theta=0$ mentioned above is indeed a minimum of the potential. ${ }^{15}$

\section{$5 \quad$ Free energy as a function of imaginary chemical potential}

In this section we will calculate the free energy of the D3-D7 system (primarily as a function of the location $t_{f}^{\prime}$ of the $\mathrm{D} 7$ branes on the temporal circle). As mentioned before, we will use the dual gravity description (2.6) for the D3 branes and consider the D7 branes as probes coupled to this geometry through a DBI action. In the deconfinement geometry (2.6), there are two possible stable configurations of the D7-branes which correspond to the chiral symmetry being preserved/broken [1] (see figure 1).

\footnotetext{
${ }^{12}$ In the holographic setup $t_{f}^{\prime}$ corresponds to the point on the $t^{\prime}$-circle reached by the D7 brane at the boundary, i.e. $t_{f}^{\prime}=\lim _{u \rightarrow \infty} t^{\prime}(u)$.

${ }^{13}$ The length of the temporal circle in the IIB is denoted by $\beta^{\prime}$ which equals $4 \pi^{2} / \beta$.

${ }^{14}$ This can be regarded as the potential energy between the D7 and D3 branes in the open string description.

${ }^{15}$ See (5.6). Other minima, corresponding to non-zero values of the integer $k$, correspond to the presence of $k$ D3-brane pairs as explained in footnote 26 .
} 
(a) chiral symmetry preserved

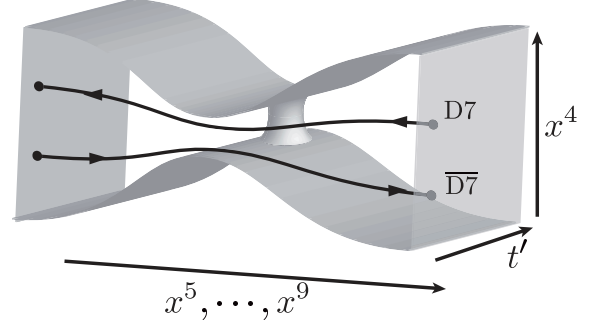

(b) chiral symmetry broken

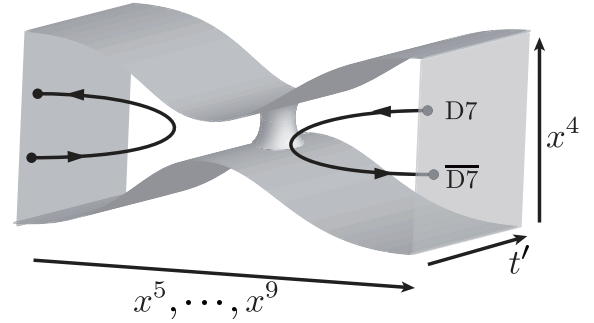

Figure 1. D7/吕-branes in the localized solitonic D3-brane background (2.6). There are two

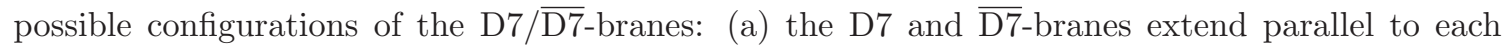
other, which implies that the chiral symmetry is preserved, (b) the D7 and $\overline{\mathrm{D} 7}$-branes are connected, which implies that the chiral symmetry is broken.

Let us consider the chiral-symmetric configuration for simplicity; it is straightforward to generalize our considerations to the broken symmetry configuration. We assume that the branes are not curved in $x_{4}$ space. Then the induced metric on the D7-branes is given by

$$
\begin{aligned}
d s_{\mathrm{D} 7}^{2} & =\alpha^{\prime}\left[H^{-1 / 2}\left(\sum_{i=1}^{3} d x_{i}^{2}\right)+H^{1 / 2}\left(\left(1+\left(\frac{d t^{\prime}(u)}{d u}\right)^{2}\right) d u^{2}+u^{2} d \Omega_{4}^{2}\right)\right], \\
H & =\sum_{n} \frac{2 \lambda_{5} / \beta}{\left(u^{2}+\left(t^{\prime}(u)-n \beta^{\prime}\right)^{2}\right)^{2}} \\
& =\frac{\lambda_{5}}{4 \pi}\left[\frac{1}{u^{3}} \operatorname{Re}\left(\frac{1}{\tanh \left(\frac{\pi\left(u-i t^{\prime}(u)\right)}{\beta^{\prime}}\right)}\right)+\frac{\pi}{\beta^{\prime} u^{2}} \operatorname{Re}\left(\frac{1}{\sinh ^{2}\left(\frac{\pi\left(u-i t^{\prime}(u)\right)}{\beta^{\prime}}\right)}\right)\right],
\end{aligned}
$$

where $t^{\prime}(u)$ is the position of the D7-brane subject to the boundary condition $t^{\prime}(u \rightarrow \infty)=$ $t_{f}^{\prime}$. We have assumed that the D7-brane is far away from the D3-brane and neglected the quantity $\Phi$ appearing in the metric (2.6). Later we will justify the validity of this assumption. The DBI action for the $N_{f}$ D7-branes then becomes

$$
\begin{aligned}
S_{\mathrm{DBI}}^{\mathrm{D} 7} & =N_{f} T_{7} \int d^{8} x e^{-\left(\phi-\phi_{\infty}\right)} \sqrt{\operatorname{det} g_{D 7}} \\
& =\frac{8 \pi^{2} N_{f} N_{c} \beta}{3(2 \pi)^{6} \lambda_{5}} V_{3} \int_{0}^{\infty} d u u^{4} H^{1 / 2}\left(1+\left(\frac{d t^{\prime}(u)}{d u}\right)^{2}\right)^{\frac{1}{2}} .
\end{aligned}
$$

Here $V_{3}$ is the volume of the $x_{1}, x_{2}, x_{3}$ space, and we have used $e^{\phi_{\infty}}=g_{s}$ and $T_{7}=$ $1 / g_{s}(2 \pi)^{7} \alpha^{\prime 4}$ which is the tension of the D7-brane. The Euclidean action has a minimum at the constant value $t^{\prime}(u)=\beta^{\prime} / 2\left(\bmod \beta^{\prime}\right) .{ }^{16}$ Thus the stable configuration of the D7branes is $t^{\prime}(u)=t_{f}^{\prime}=\beta^{\prime} / 2$ with $d t^{\prime}(u) / d u=0$. This configuration is precisely (4.4) which corresponds to $\theta=0 .{ }^{17}$

\footnotetext{
${ }^{16}$ To prove this, note that the minimization of the action requires (a) setting $d t^{\prime} / d u=0$ and (b) minimizing $H$. From the second line of $(5.1), H$ for constant $t^{\prime}(u)=t_{f}^{\prime}$ is clearly minimized at $t_{f}^{\prime}=\beta^{\prime} / 2\left(\bmod \beta^{\prime}\right)$.

${ }^{17}$ Ref. [1] investigated the chiral symmetry breaking/restoration transition by comparing the DBI action for the configurations of the D7-branes corresponding to these phases (see figure 1). In this study, the D7-
} 
To calculate the $t_{f}^{\prime}$ dependence of the DBI action, we approximate $d t^{\prime}(u) / d u=0,{ }^{18}$ and take $t^{\prime}(u)=t_{f}^{\prime}$. The integral (5.2) bceomes as

$$
\int_{0}^{\infty} d u u^{4} H^{1 / 2}=\frac{1}{\beta^{3}} \sqrt{\frac{2 \lambda_{5}}{\beta}}\left(c_{0}+c_{2}\left(\beta t_{f}^{\prime}-2 \pi^{2}\right)^{2}+\cdots\right),
$$

where $c_{0}$ is a divergent constant which should be removed through a regularization and $c_{2}=20.7022 \ldots$ This gives us the potential of a single D7 brane as a function of the position variable $t_{f}^{\prime}$. As we found in section $4, t_{f}^{\prime}$ is directly related to the imaginary chemical potential $\theta$, according to the relation (4.3). By substituting this relation into the above and summing up the contributions of all D7 and $\overline{\mathrm{D} 7}$-branes, we obtain the $\theta$ dependent classical action

$$
S_{\mathrm{DBI}}^{\mathrm{D} 7 \overline{\mathrm{D} 7}}=\frac{2 N_{c} N_{f} V_{3}}{3 \pi^{2} \sqrt{\lambda_{Y M} L_{4}}} T^{\frac{5}{2}} c_{2} \theta^{2}+\cdots
$$

Once again, we see that the choice $\theta=0$, made in [1], and given by (4.4) is a consistent classical solution according to this potential.

This, however, is not the end of the story. The partition function of QCD with an imaginary chemical potential (3.2) has a discrete symmetry

$$
Z(T ; \theta)=Z\left(T ; \theta+2 \pi / N_{c}\right),
$$

which is a generalization of the $\mathbf{Z}_{N_{c}}$ symmetry of pure Yang-Mills theory to QCD (see appendix A.4). The potential (5.4) clearly does not respect this symmetry. To fix this problem, we need to understand how the above $\mathbf{Z}_{N_{c}}$ symmetry is realized in the gravity dual. We find, following similar considerations in [27-31], that the missing pieces of the puzzle are provided by the dynamics of some bulk gauge fields, namely, in our setup, the graviphoton (KK gauge field) and $C_{4}$ gauge field. We give the details in appendix B; the upshot is that the dynamics of these gauge fields changes the classical action (5.4) into

$$
S(\theta)=\frac{2 N_{c} N_{f} V_{3}}{3 \pi^{2} \sqrt{\lambda_{Y M} L_{4}}} T^{\frac{5}{2}}\left[c_{2} \min _{k \in \mathbf{Z}}\left(\theta-\frac{2 \pi k}{N_{c}}\right)^{2}+\cdots\right] .
$$

The above action, plotted in figure 2 , obviously has the discrete symmetry $\theta \rightarrow \theta+2 \pi / N_{c}$. The potential has $N_{c}$ minima for $\theta(0 \leq \theta<2 \pi)$ and indicates $N_{c}$ phase transitions as $\theta$ varies. Note that the minimum discussed above, namely $\theta=0$ (corresponding to (4.4)) remains a minimum for the above potential as well. Through each transition, the distance between the D7-branes and the D3-branes is shifted by $\beta^{\prime} / N_{c},{ }^{19}$ and they are always antipodal at the minima of the potential. It ensures the assumption below (5.1).

branes are set at $t^{\prime}=\beta^{\prime} / 2$, since this is stable for both chiral symmetry preserved and broken configurations. Through the relation (4.3), it corresponds to $\theta=0$. This shows that the results in [1] are valid for the standard thermal quarks.

${ }^{18}$ If we do not use the approximation $d t^{\prime}(u) / d u=0, c_{2}$ is modified but qualitative properties would not change.

${ }^{19}$ To be more precise, through each transition, $\eta$ in (B.14) is shifted by $1 / N_{c}$, and hence $t_{f}^{\prime}$ is shifted by $\beta^{\prime} / N_{c}$ due to the relation above (B.13). 


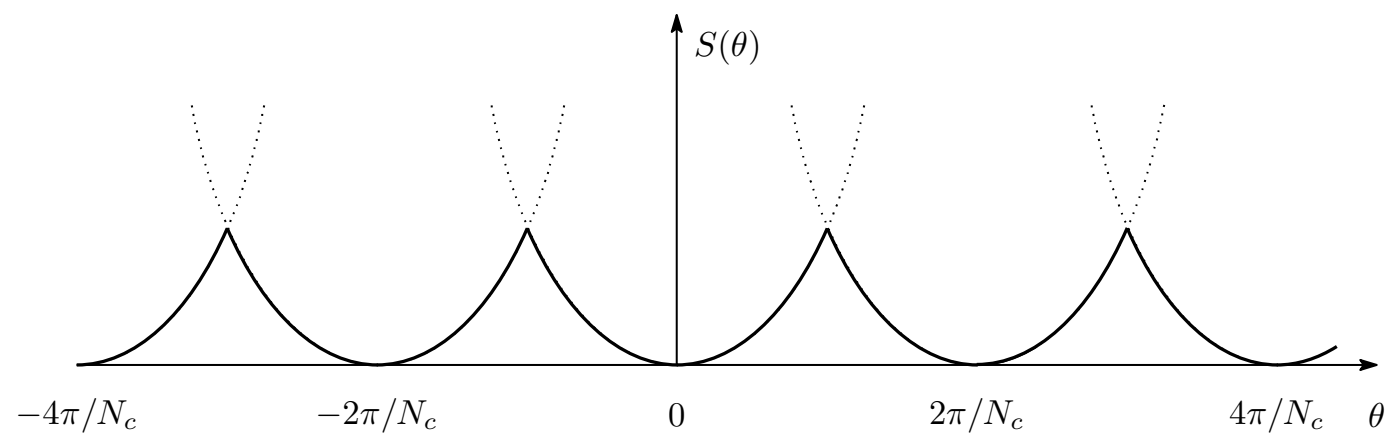

Figure 2. A schematic plot of $S(\theta)$, the effective potential for the imaginary chemical potential $\theta$.

In [22], Roberge and Weiss obtained the following result for the potential for an imaginary chemical potential $\theta$ in the deconfinement phase from perturbative QCD:

$$
V_{\text {pert. }}(\theta)=\min _{k \in \mathbf{Z}}\left[-\frac{1}{12} \pi^{2} T^{4} N_{c} N_{f}\left(1-\frac{1}{\pi^{2}}\left(\theta-\frac{2 \pi k}{N_{c}}\right)^{2}\right)^{2}\right] .
$$

Remarkably, this potential qualitatively agrees with the holographic result (5.6) we found above.

\section{Acknowledgments}

The authors would like to thank Yoshimasa Hidaka for discussions about imaginary chemical potential. The work of H. I. is supported by Iwanami Fujukai. The work of T. M. is supported in part by Grant-in-Aid for Scientific Research (No. 15K17643) from JSPS.

\section{A A review of imaginary chemical potentials in field theory}

We summarize properties of the imaginary chemical potential which are used in the main part.

\section{A.1 Relation between imaginary chemical potential and periodicity of fermions}

First we discuss the connection between the imaginary chemical potential and the periodicity of the fermions. In general, for a system described by a Hamiltonian $\widehat{H}$ with fermions described by dynamical variables $\widehat{\eta}_{i}$ (where the index $i$ refers to any set of indices of the dynamical variables including the coordinates), we can derive the following identity

$$
\begin{aligned}
& \operatorname{tr}\left(e^{-\beta \widehat{H}+i \theta \widehat{N}}\right) \\
& \quad=\int_{\vec{\eta}(\beta)=-\vec{\eta}(0)}\left[d \vec{\eta}^{*} d \vec{\eta}\right] \exp \left\{-\int_{0}^{\beta} d t\left[\sum_{i} \eta_{i}{ }^{*} \partial_{t} \eta_{i}+H\left(\vec{\eta}^{*}, \vec{\eta}\right)-i \beta^{-1} \theta \sum_{i} \eta_{i}{ }^{*} \eta_{i}\right]\right\} \\
& \quad=\int_{\vec{\eta}(\beta)=-e^{-i \theta} \vec{\eta}(0)}\left[d \vec{\eta}^{*} d \vec{\eta}\right] \exp \left\{-\int_{0}^{\beta} d t\left[\sum_{i} \eta_{i}{ }^{*} \partial_{t} \eta_{i}+H\left(\vec{\eta}^{*}, \vec{\eta}\right)\right]\right\}
\end{aligned}
$$


where $t$ is the Euclidean time and $\widehat{N}:=\sum_{i} \widehat{\eta}_{i}{ }^{*} \widehat{\eta}_{i}$ is the fermion number operator. The trace on the left hand side is taken over the fermions. We can derive this relation in a usual manner with the slicing in $\beta$-direction and the coherent state representation. The nontrivial periodicity on the second line results from the identity $e^{i \theta \widehat{N}}|\vec{\eta}\rangle=\left|e^{i \theta} \vec{\eta}\right\rangle$ on the coherent state $|\vec{\eta}\rangle$ in the trace. Therefore we can identify the imaginary chemical potential with the monodromy of the fermions around the Euclidean time circle. ${ }^{20}$

\section{A.2 Relation between imaginary chemical potential and gauge fields}

Next we establish the connection between the imaginary chemical potential and the $\mathrm{U}(1)$ component of an external gauge field. Let us consider a QCD-like theory with a color gauge group $G_{c}$ and matter fermions $\psi$ in the fundamental representation of $G_{c}$ and some representation of a flavor group $G_{f}$. We gauge both $G_{c}$ and $G_{f}$ with the gauge potentials $A^{c}$ and $A^{f}$ respectively, and regard $A^{c}$ as dynamical and $A^{f}$ as external. The Lagrangian density (in the Lorentzian signature) is

$$
\mathcal{L}_{m}=-\bar{\psi}\left(\gamma^{\mu} D_{\mu}+m\right) \psi
$$

with the following definitions:

$$
\bar{\psi}:=\psi^{\dagger} i \gamma^{0}, \quad D_{\mu} \psi:=\partial_{\mu} \psi-i A_{\mu}^{c} \psi-i A_{\mu}^{f} \psi, \quad\left\{\gamma^{\mu}, \gamma^{\nu}\right\}=2 \eta^{\mu \nu}, \quad \eta_{\mu \nu}=\operatorname{diag}(-1,1,1,1) .
$$

The Hamiltonian reads

$$
H_{m}\left[A^{f}\right]=\int d^{3} x\left[-\psi^{\dagger} A_{0} \psi+\bar{\psi} \gamma^{i} D_{i} \psi+m \bar{\psi} \psi\right]
$$

where $A:=A^{c}+A^{f}$, and we displayed the external flavor field $A^{f}$ explicitly in the Hamiltonian. Note that the index 0 stands for the Lorentzian time while $t$ stands for the Euclidean time.

Now let us turn on only the temporal component $A_{0}^{f}$ of the external flavor gauge field $A^{f}$ and suppose that this $A_{0}^{f}$ is proportional to the identity matrix. Then the first equality of (A.1) with the Hamiltonian $H_{m}\left[A^{f}\right]$ and the number operator $\widehat{N}:=\int d^{3} x \psi^{\dagger} \psi$ reads

$$
\begin{aligned}
& \operatorname{tr}\left(\exp \left[-\beta \widehat{H}\left[A_{0}^{f}\right]+i \theta \widehat{N}\right]\right) \\
& =\int_{\psi(\beta)=-\psi(0)}\left[d A^{c} d \psi^{*} d \psi\right] e^{-S_{c}\left[A^{c}\right]} \\
& \quad \times \exp \left\{-\int_{0}^{\beta} d t d^{3} x\left[\psi^{\dagger} \partial_{t} \psi+\bar{\psi}\left(\gamma^{i} D_{i}+m\right) \psi-i \psi^{\dagger} A_{t} \psi-i \beta^{-1} \theta \psi^{\dagger} \psi\right]\right\}
\end{aligned}
$$

\footnotetext{
${ }^{20}$ Two known special cases of (A.1) are worth noting: (a) a thermal partition function with no imaginary chemical potential $(\theta=0)$ corresponds to an antiperiodic boundary condition around the Euclidean time circle, and (b) a thermal partition function with an imaginary chemical potential $\theta=\pi$ corresponds to the periodic boundary condition. The latter observation ties up with the fact that an insertion of $e^{i \theta \hat{N}}$ in the l.h.s. of (A.1) with $\theta=\pi$ is equivalent to inserting $(-1)^{\hat{N}}$, which, of course, converts a thermal partition function into a Witten index, and is hence represented by a path integral over periodic fermions.
} 


$$
\begin{aligned}
&=\int_{\psi(\beta)=} {\left[d A^{c} d \psi^{*} d \psi\right] e^{-S_{c}\left[A^{c}\right]} } \\
& \times \exp \left\{-\int_{0}^{\beta} d t d^{3} x\left[\psi^{\dagger} \partial_{t} \psi+\bar{\psi}\left(\gamma^{i} D_{i}+m\right) \psi-i \psi^{\dagger} \beta^{-1}\left(\beta A_{t}+\theta\right) \psi\right]\right\} \\
&=\operatorname{tr}\left(\exp \left[-\beta \widehat{H}[0]+i\left(\theta+\beta A_{t}^{f}\right) \widehat{N}\right]\right)
\end{aligned}
$$

where we defined $A_{t}:=-i A_{0}=A^{c}{ }_{t}+A^{f}{ }_{t}$, and $S_{c}\left[A^{c}\right]$ is the Euclideanized Yang-Mills action of the color gauge field. ${ }^{21}$ The final expression makes it clear that $A_{t}^{f}$ (times $\beta$ ) plays the same role of an imaginary chemical potential $\theta$.

\section{A.3 Derivation of (4.1)}

We will find a string-theoretical setup which yields the thermal partition function with an imaginary chemical potential. The thermal partition function is given by a path integral of a Euclideanized QCD-like theory with anti-periodic fermions while a gauge theory on the D4/D8/ $\overline{\mathrm{D} 8}$ branes in our string-theoretical setup is a Euclidean QCD-like theory with periodic fermions. If we add a diagonal gauge field $A_{t}$ on $\mathrm{D} 8 / \overline{\mathrm{D} 8}$, this couples with the fermions as a flavor gauge field. Thus the string theory side gives us the following path integral $^{22}$

$$
\int_{\psi(\beta)=\psi(0)}\left[d A^{c} d \psi^{*} d \psi\right] e^{-S_{c}\left[A^{c}\right]} \exp \left\{-\int_{0}^{\beta} d t d^{3} x\left[\psi^{\dagger} \partial_{t} \psi+\bar{\psi}\left(\gamma^{i} D_{i}+m\right) \psi-i \psi^{\dagger} \beta^{-1} \beta A_{t} \psi\right]\right\} .
$$

By the second equality of (A.1), this path integral is equal to

$$
\begin{aligned}
\int_{\psi(\beta)} & {\left[d A^{c} d \psi^{*} d \psi\right] e^{-S_{c}\left[A^{c}\right]} } \\
& \times \exp \left\{-\int_{0}^{\beta} d t d^{3} x\left[\psi^{\dagger} \partial_{t} \psi+\bar{\psi}\left(\gamma^{i} D_{i}+m\right) \psi-i \psi^{\dagger} \beta^{-1}\left(\beta A_{t}-\pi\right) \psi\right]\right\} .
\end{aligned}
$$

This is equal to the thermal partition function with an imaginary chemical potential $\beta A_{t}-\pi$. In other words, if we want to compute a thermal partition function with an imaginary chemical potential $\theta$, then we may consider a $\mathrm{D} 4 / \mathrm{D} 8 / \overline{\mathrm{D} 8}$-brane setup with a diagonal gauge field $\beta^{-1}(\theta+\pi)$ on the $\mathrm{D} 8 / \overline{\mathrm{D}} 8$-branes.

\section{A.4 Periodicity of thermal partition function with imaginary chemical poten- tial}

Finally, we explain the periodicity (5.5) of the partition function $Z(T, \theta):=\operatorname{tr}\left(e^{-\beta \widehat{H}+i \theta \widehat{N}}\right)$, where $G_{c}=\mathrm{SU}\left(N_{c}\right)$ and $G_{f}=\mathrm{U}\left(N_{f}\right)$. Its path integral expression can be written down

\footnotetext{
${ }^{21}$ The trace is taken not only over the matter fermions but also over the color gauge field.

${ }^{22}$ More precisely, a classical supergravity plus the D8/D8-brane actions evaluated at an on-shell configuration gives us (A.5) of the corresponding gauge theory, which is a QCD-like theory with periodic fermions. We perform this in the T-dual setup in section 5 .
} 
using (A.2) as

$$
\begin{aligned}
Z(T, \theta) & =\int_{\psi(\beta)=-e^{-i \theta} \psi(0)}\left[d A^{c} d \psi^{*} d \psi\right] e^{-S\left[\psi, \bar{\psi}, A^{c}\right]-S_{c}\left[F^{c}\right]}, \\
S\left[\psi, \bar{\psi}, A^{c}\right]: & =\int_{0}^{\beta} d t d^{3} x\left[\psi^{\dagger} \partial_{t} \psi+\bar{\psi}\left(\gamma^{i} D_{i}+m\right) \psi-i \psi^{\dagger} A_{t}^{c} \psi\right] .
\end{aligned}
$$

Let us consider the following $\mathrm{SU}\left(N_{c}\right)$ transformation $g$ with a $\mathbf{Z}_{N_{c}}$-twisted boundary condition

$$
g(\beta, \boldsymbol{x})=e^{-2 \pi i k / N_{c}} g(0, \boldsymbol{x}), \quad g(t, \boldsymbol{x}) \in \mathrm{SU}\left(N_{c}\right), \quad k=0,1, \cdots, N_{c}-1 .
$$

Under this transformation, the fermions $\psi$ and the Polyakov loop $W\left(A^{c}\right)$ transform as

$$
\psi(t, \boldsymbol{x}) \mapsto \psi^{g}(t, \boldsymbol{x})=g(t, \boldsymbol{x}) \psi(t, \boldsymbol{x}), \quad W\left(A^{c}\right) \mapsto W\left(A^{c g}\right)=e^{-2 \pi i k / N_{c}} W\left(A^{c}\right) .
$$

This transformation changes the periodicity of the matter fields as follows

$$
\psi^{g}(\beta, \boldsymbol{x})=-e^{-i \theta} \psi(0, \boldsymbol{x}) \longrightarrow \psi^{g}(\beta, \boldsymbol{x})=-e^{-i\left(\theta+2 \pi k / N_{c}\right)} \psi^{g}(0, \boldsymbol{x}) .
$$

Then we can derive the periodicity (5.5) as follows:

$$
\begin{aligned}
Z(T, \theta) & =\int_{\psi(\beta)=-e^{-i \theta} \psi(0)}\left[d A^{c} d \psi^{*} d \psi\right] e^{-S[\psi, \bar{\psi}, A]-S_{c}\left[F^{c}\right]} \\
& =\int_{\psi^{g}(\beta)=-e^{-i\left(\theta+2 \pi k / N_{c}\right)} \psi^{g}(0)}\left[d A^{c g} d \psi^{* g} d \psi^{g}\right] e^{-S\left[\psi^{g}, \bar{\psi}^{g}, A^{c g}\right]-S_{c}\left[F^{c g}\right]} \\
& =Z\left(T, \theta+2 \pi k / N_{c}\right),
\end{aligned}
$$

where in the second equality we used the invariance of the path integral measure and the action under $g$.

\section{B $\mathrm{Z}_{N_{c}}$ symmetry for $\theta$ in gravity}

In this section, we show how the discrete $\mathbf{Z}_{N_{c}}$ symmetry $\theta \rightarrow \theta+2 \pi / N_{c}$ discussed around (5.5) is realized in the localized D3-brane system. The origin of this symmetry, as noted above, is the fact that the color gauge group dual to the near horizon geometry is $\mathrm{SU}\left(N_{c}\right)$ (rather than $\mathrm{U}\left(N_{c}\right)$ ) with a center $\mathbf{Z}_{N_{c}}$. Related symmetries in black brane geometries have been discussed in $[28,31]$, and our derivation is similar to these works.

To see the $\mathbf{Z}_{N_{c}}$ symmetry in type IIB supergravity, we consider a Kaluza-Klein decomposition on the $t^{\prime}$-cycle and study the low energy dynamics in terms of the 9 dimensional IIB supergravity. The $9 \mathrm{~d}$ supergravity action has the following terms ${ }^{23}$

$$
\frac{\beta^{\prime}}{(2 \pi)^{7} \alpha^{\prime 4}}\left(\frac{1}{2} \int d^{9} x \sqrt{g} F_{4}^{2}+\int A_{1} \wedge F_{4} \wedge F_{4}\right)
$$

\footnotetext{
${ }^{23}$ These are related, by T-duality along the $t^{\prime}$-cycle, to the terms

$$
\frac{1}{2} \int d^{10} x \sqrt{g} F_{4}^{2}+\int B_{2} \wedge F_{4} \wedge F_{4}
$$

in IIA supergravity which are employed in [31] to explore the $\mathbf{Z}_{N_{c}}$ symmetry in the black D4-brane background.
} 
where $F_{4}=d C_{3}$, the three-form potential $C_{3}$ is the dimensional reduction of the four-form potential $C_{4}$, and the one-form potential $A_{1}$ is the KK gauge field from the 10 dimensional metric. We will show that

$$
\eta \equiv \frac{1}{\beta^{\prime}} \int_{u_{0}}^{\infty} A_{u} d u
$$

takes a discrete value $k / N_{c}$ for some integer $k$ at low energy - a fact which, we will see, reflects the $\mathbf{Z}_{N_{c}}$ symmetry. ${ }^{24}$

At low energies, the kinetic term of (B.1) reduces to

$$
\frac{1}{2 e^{2}} \int_{\mathbf{R}^{3} \times S_{L_{4}}^{1}} d^{4} x F_{4}^{2}
$$

through the integration over $S^{4}$ and the $u$ direction. Here $e^{2}$ is an effective coupling. ${ }^{25}$ The CS term of (B.1) becomes

$$
\begin{aligned}
\frac{\beta^{\prime}}{(2 \pi)^{7} \alpha^{\prime 4}} \int A_{1} \wedge F_{4} \wedge F_{4} & =\frac{1}{\beta^{\prime}} \int d u A_{u} \frac{\beta^{\prime}}{(2 \pi)^{4} \alpha^{\prime 2}} \int_{S^{4}} F_{4} \frac{\beta^{\prime}}{(2 \pi)^{3} \alpha^{\prime 2}} \int_{\mathbf{R}^{3} \times S_{L_{4}}^{1}} F_{4} \\
& =\mu_{3} \beta^{\prime} \eta N_{c} \int_{\mathbf{R}^{3} \times S_{L_{4}}^{1}} F_{4}
\end{aligned}
$$

where we have used $\frac{\beta^{\prime}}{(2 \pi)^{4} \alpha^{\prime 2}} \int_{S^{4}} F_{4}=N_{c}$ and $\mu_{3}:=(2 \pi)^{-3} \alpha^{\prime-2}$ is the tension of a D3-brane. Therefore the Hamiltonian density from the action (B.1) reads

$$
\mathcal{H}=\frac{e^{2}}{2}\left(\Pi-\mu_{3} \beta^{\prime} \eta N_{c}\right)^{2},
$$

where $\Pi:=F_{4} / e^{2}+\mu_{3} \eta N_{c}=-i \delta / \delta C_{3}$ is the conjugate momentum of $C_{3}$ with regard to one of the three directions $\left(x_{1}, x_{2}, x_{3}\right)$ of $\mathbf{R}^{3}$ (see (2.4)) as the 'time' direction for the canonical quantization. Then similarly to 2d QED [32,33], a basis of energy eigenstates of this system can be taken as $[28,31]$

$$
\Psi_{k}=\exp \left(i k \beta^{\prime} \mu_{3} \int_{R^{2} \times S_{L_{4}}^{1}} C_{3}\right),
$$

with energy eigenvalue

$$
\mathcal{H} \Psi_{k}=\frac{e^{2} \mu_{3}^{2} \beta^{\prime 2}}{2}\left(k-\eta N_{c}\right)^{2} \Psi_{k}
$$

\footnotetext{
${ }^{24} u_{0}$ denotes the IR end of $u$-coordinate in the 9 dimensional theory which is related to the position of the horizon of the localized D3-brane geometry. Although the position of the horizon depends on $t^{\prime}$ and $u$ in the original 10 dimensional system, within the KK-decomposition the $t^{\prime}$ dependence of the metric is mapped to the condensation of KK non-zero modes.

${ }^{25}$ Although $u$ integral is over an infinite range, the effective coupling $e^{2}$ is finite, as in the case of a similar discussion for black branes [28, 31].
} 
Here $k$ must be integer-quantized. Note that under a shift $\eta \rightarrow \eta+1 / N_{c}$, there is a monodromy $\Psi_{k} \rightarrow \Psi_{k+1}$ accompanying a pair creation of D3-branes ${ }^{26}$ (this is an analogue of a pair creation of the electrons in 2d QED [32]). Therefore the potential for $\eta$ becomes

$$
V(\eta)=\min _{k \in \mathbf{Z}} \frac{e^{2} \mu_{3}^{2} \beta^{\prime 2}}{2}\left(k-\eta N_{c}\right)^{2}
$$

and is minimized at $\eta=k / N_{c}$ for some integer $k$. Hence $\eta$ is restricted to discrete values at low energy.

Now let us show that the shift $\eta \rightarrow \eta+1 / N_{c}$ induces the $\mathbf{Z}_{N_{c}}$ symmetry for $\theta$. We have considered the 9 dimensional supergravity so far and ignored the KK non-zero modes. However they should be included in the system, and importantly they always couple to the KK gauge field $A_{u}$ through the covariant derivative

$$
\left(\partial_{u}-i \frac{2 \pi n}{\beta^{\prime}} A_{u}\right) \phi_{n}
$$

where $\phi_{n}$ denotes the $n$-th KK mode of field $\phi\left(=\sum_{n} e^{2 \pi i n t^{\prime} / \beta^{\prime}} \phi_{n}\right)$ which symbolically denotes all fields in the nine dimensional supergravity except $A_{u}$. Now $A_{u}$ can be absorbed into the phases of the KK modes by the field redefinition

$$
\phi_{n}=\exp \left(i \frac{2 \pi n}{\beta^{\prime}} \int_{u_{0}}^{u} d u^{\prime} A_{u}\right) \tilde{\phi}_{n} .
$$

Using this fact, we show that we can read off the solution of supergravity with non-zero $A_{u}$ from the solution with $A_{u}=0$. Since $A_{u}$ with no derivatives appears only at the CS term (B.1) after the field redefinition (B.11), the equation of motion for $\tilde{\phi}_{n}$ is the same as one in $A_{u}=0$ case except for the RR fields which couple to $A_{u}$ directly through the CS coupling. Thus if we ignore the backreaction of these RR fields, $\tilde{\phi}_{n}=\left.\phi_{n}\right|_{A_{u}=0}$ is a solution of supergravity with $A_{u} \neq 0$, where $\left.\phi_{n}\right|_{A_{u}=0}$ is a solution of supergravity with $A_{u}=0$. Therefore in terms of the original ten dimensional coordinates

$$
\phi\left(t^{\prime}, u\right)=\left.\sum_{n} \exp \left(i \frac{2 \pi n t^{\prime}}{\beta^{\prime}}+i \frac{2 \pi n}{\beta^{\prime}} \int_{u_{0}}^{u} d u^{\prime} A_{u}\right) \phi_{n}\right|_{A_{u}=0} .
$$

is a solution of the supergravity with non-zero $A_{u}$. It means that the solution is obtained just by replacing $t^{\prime} \rightarrow t^{\prime}+\int_{u_{0}}^{u} d u^{\prime} A_{u}$.

Let us consider how the free energy for the imaginary chemical potential (5.4) is modified by $A_{u}$. The free energy depends on $\theta$ which is related to the position of the D7-branes

\footnotetext{
${ }^{26}$ The brane configuration of the created D3-branes are given by

$$
\begin{aligned}
& \left(t^{\prime}\right) \quad 1 \quad 2 \quad 3 \quad(4) 566789 \\
& N_{c} \text { D3-brane (LSD3) } \quad--- \\
& \text { pairs of D3-branes } \quad-\quad-\quad-
\end{aligned}
$$

where we have taken $x^{3}$ as the 'time' direction for the canonical quantization. Note that this brane configuration is the T-dual of the black D4-brane system studied in [31].
} 
at the boundary $(u \rightarrow \infty)$ via (4.3). Thus according to the above arguments, we replace $t^{\prime} \rightarrow t^{\prime}+\int_{u_{0}}^{\infty} d u A_{u}=t^{\prime}+\beta^{\prime} \eta$ (and hence $\theta \rightarrow \theta+2 \pi \eta$ by (4.3)) to obtain

$$
S_{\mathrm{DBI}}^{\mathrm{D} 7 \overline{\mathrm{D} 7}}=\frac{2 N_{c} N_{f} V_{3}}{3 \pi^{2} \sqrt{\lambda_{Y M} L_{4}}} T^{\frac{5}{2}} c_{2}(\theta+2 \pi \eta)^{2} .
$$

By adding the energy (B.9) for $F_{4}$, we obtain the $\eta$-dependent terms of the classical action

$$
S_{\mathrm{DBI}}^{\mathrm{D} 7 \overline{\mathrm{D} 7}}+S_{F_{4}}=\frac{2 N_{c} N_{f} V_{3}}{3 \pi^{2} \sqrt{\lambda_{Y M} L_{4}}} T^{\frac{5}{2}} c_{2}(\theta+2 \pi \eta)^{2}+\min _{k \in \mathbf{Z}} \frac{L_{4} V_{3} e^{2} \mu_{3}^{2} \beta^{\prime 2}}{2}\left(k-\eta N_{c}\right)^{2} .
$$

Then after an integral over $\eta$ the system acquires the symmetry $\theta \rightarrow \theta+2 \pi / N_{c}$ by compensating the shift of $\eta$ as expected in QCD (5.5). By regarding this symmetry, we can rewrite the $\theta$-dependent part of the classical action (B.14) $\mathrm{as}^{27}$

$$
S(\theta)=\frac{2 N_{c} N_{f} V_{3}}{3 \pi^{2} \sqrt{\lambda_{Y M} L_{4}}} T^{\frac{5}{2}} c_{2} \min _{k \in \mathbf{Z}}\left(\theta-\frac{2 \pi k}{N_{c}}\right)^{2} .
$$

Thus we obtain (5.6).

Open Access. This article is distributed under the terms of the Creative Commons Attribution License (CC-BY 4.0), which permits any use, distribution and reproduction in any medium, provided the original author(s) and source are credited.

\section{References}

[1] G. Mandal and T. Morita, Gregory-Laflamme as the confinement/deconfinement transition in holographic QCD, JHEP 09 (2011) 073 [arXiv:1107.4048] [INSPIRE].

[2] E. Witten, Anti-de Sitter space, thermal phase transition and confinement in gauge theories, Adv. Theor. Math. Phys. 2 (1998) 505 [hep-th/9803131] [InSPIRE].

[3] T. Sakai and S. Sugimoto, Low energy hadron physics in holographic QCD, Prog. Theor. Phys. 113 (2005) 843 [hep-th/0412141] [INSPIRE].

[4] J.M. Maldacena, The large- $N$ limit of superconformal field theories and supergravity, Int. J. Theor. Phys. 38 (1999) 1113 [Adv. Theor. Math. Phys. 2 (1998) 231] [hep-th/9711200] [INSPIRE].

[5] Y. Kim, I.J. Shin and T. Tsukioka, Holographic QCD: past, present and future, Prog. Part. Nucl. Phys. 68 (2013) 55 [arXiv: 1205.4852] [InSPIRE].

[6] A. Rebhan, The Witten-Sakai-Sugimoto model: a brief review and some recent results, EPJ Web Conf. 95 (2015) 02005 [arXiv: 1410.8858] [INSPIRE].

[7] O. Aharony, J. Sonnenschein and S. Yankielowicz, A holographic model of deconfinement and chiral symmetry restoration, Annals Phys. 322 (2007) 1420 [hep-th/0604161] [INSPIRE].

[8] O. Aharony, S.S. Gubser, J.M. Maldacena, H. Ooguri and Y. Oz, Large-N field theories, string theory and gravity, Phys. Rept. 323 (2000) 183 [hep-th/9905111] [INSPIRE].

\footnotetext{
${ }^{27}$ We performed an integral over $\eta$, observing that the coefficient of $\eta^{2}$ in the second term of (B.14) is much larger by $N_{c}$ than that in the first term. In addition, the value of the obtained classical action (B.15) is $O\left(N_{c}\right)$, and we can ignore the back reaction of these fields to the background geometry.
} 
[9] K.-Y. Kim, S.-J. Sin and I. Zahed, Dense hadronic matter in holographic QCD, J. Korean Phys. Soc. 63 (2013) 1515 [hep-th/0608046] [INSPIRE].

[10] N. Horigome and Y. Tanii, Holographic chiral phase transition with chemical potential, JHEP 01 (2007) 072 [hep-th/0608198] [INSPIRE].

[11] S.-J. Sin, Gravity back-reaction to the baryon density for bulk filling branes, JHEP 10 (2007) 078 [arXiv:0707.2719] [INSPIRE].

[12] D. Yamada, Sakai-Sugimoto model at high density, JHEP 10 (2008) 020 [arXiv:0707.0101] [INSPIRE].

[13] O. Bergman, G. Lifschytz and M. Lippert, Holographic Nuclear Physics, JHEP 11 (2007) 056 [arXiv:0708.0326] [INSPIRE].

[14] M. Rozali, H.-H. Shieh, M. Van Raamsdonk and J. Wu, Cold nuclear matter in holographic QCD, JHEP 01 (2008) 053 [arXiv: 0708.1322] [INSPIRE].

[15] T. Eguchi and H. Kawai, Reduction of dynamical degrees of freedom in the large- $N$ gauge theory, Phys. Rev. Lett. 48 (1982) 1063 [InSPIRE].

[16] A. Gocksch and F. Neri, On large-n QCD at finite temperature, Phys. Rev. Lett. 50 (1983) 1099 [INSPIRE].

[17] K. Dasgupta, C. Gale, M. Mia, M. Richard and O. Trottier, Infrared dynamics of a large- $N$ QCD model, the massless string sector and mesonic spectra, JHEP 07 (2015) 122 [arXiv: 1409.0559] [INSPIRE].

[18] F. Chen, L. Chen, K. Dasgupta, M. Mia and O. Trottier, Ultraviolet complete model of large- $N$ thermal QCD, Phys. Rev. D 87 (2013) 041901 [arXiv: 1209.6061] [INSPIRE].

[19] T. Azuma, T. Morita and S. Takeuchi, Hagedorn instability in dimensionally reduced large- $N$ gauge theories as Gregory-Laflamme and Rayleigh-Plateau instabilities, Phys. Rev. Lett. 113 (2014) 091603 [arXiv:1403.7764] [inSPIRE].

[20] T. Morita, S. Shiba, T. Wiseman and B. Withers, Moduli dynamics as a predictive tool for thermal maximally supersymmetric Yang-Mills at large-N, JHEP 07 (2015) 047 [arXiv: 1412.3939] [INSPIRE].

[21] M. Hanada, Y. Matsuo and T. Morita, Instanton dynamics in finite temperature QCD via holography, Nucl. Phys. B 899 (2015) 631 [arXiv: 1505.04498] [InSPIRE].

[22] A. Roberge and N. Weiss, Gauge theories with imaginary chemical potential and the phases of QCD, Nucl. Phys. B 275 (1986) 734 [inSPIRE].

[23] K. Kashiwa and A. Ohnishi, Topological feature and phase structure of QCD at complex chemical potential, Phys. Lett. B 750 (2015) 282 [arXiv:1505.06799] [INSPIRE].

[24] N. Itzhaki, J.M. Maldacena, J. Sonnenschein and S. Yankielowicz, Supergravity and the large- $N$ limit of theories with sixteen supercharges, Phys. Rev. D 58 (1998) 046004 [hep-th/9802042] [INSPIRE].

[25] A.M. Polyakov, Gauge fields and strings, Contemporary Concepts in Physics volume 3, CRC Press, U.S.A. (1987).

[26] R. Gregory and R. Laflamme, The instability of charged black strings and p-branes, Nucl. Phys. B 428 (1994) 399 [hep-th/9404071] [INSPIRE].

[27] J. Rafferty, Holographic Roberge Weiss transitions II - Defect theories and the Sakai-Sugimoto model, JHEP 09 (2011) 087 [arXiv: 1103.2315] [INSPIRE].

[28] O. Aharony and E. Witten, Anti-de Sitter space and the center of the gauge group, JHEP 11 (1998) 018 [hep-th/9807205] [INSPIRE]. 
[29] E. Witten, AdS/CFT correspondence and topological field theory, JHEP 12 (1998) 012 [hep-th/9812012] [INSPIRE].

[30] J.M. Maldacena, G.W. Moore and N. Seiberg, D-brane charges in five-brane backgrounds, JHEP 10 (2001) 005 [hep-th/0108152] [INSPIRE].

[31] H.-U. Yee, Fate of $Z(N)$ domain wall in hot holographic QCD, JHEP 04 (2009) 029 [arXiv: 0901.0705] [INSPIRE].

[32] S.R. Coleman, More about the massive Schwinger model, Annals Phys. 101 (1976) 239 [INSPIRE].

[33] E. Witten, $\theta$ vacua in two-dimensional quantum chromodynamics, Nuovo Cim. A 51 (1979) 325 [INSPIRE]. 